\title{
New Approaches for Task Classification about Standardization Skills
}

\author{
Toshiaki Kurokawa \\ ICES Founder, independent design thinker, toshiakikr@gmail.com \\ Received: 25 June, 2013; Accepted: 21 August, 2013

\begin{abstract}
Japanese experts group has developed a set of skill standards for standardization professionals [1]. However, the approach adopted for classifying the tasks of standardization professionals is a traditional and limited one which does not cover the whole range of activities performed by standardization professionals, and moreover neglected some of the important skills needed for people who are dedicated to standardization. In this paper, the author will try to sketch new and alternative approaches for task classification about standardization skills. One important aspect is the Standardization for Public, the other aspect is the evolutional stage of standards proposed by Ken Krechmer [2].
\end{abstract}

Keywords: Skill standard, skills for standardization professionals, task classification, standardization for public, evolutional stages of standards.

\section{Introduction}

Standards and standardization mean various things for various people. However, the importance of standards and standardization is well recognized these days. For example, recent APEC textbook on standards and standardization [6] refers various important cases for standardization and the merits of standardization. The major benefits are described for for-profit corporations so is the motive for recent development of skill standards for standardization professionals developed in Japan supported by Japanese Ministry of Economy, Trade and Industry (METI) [1].

Journal of ICT, Vol. $3 \& 4,287-300$.

doi: 10.13052/jicts2245-800X.132

(c) 2014 River Publishers. All rights reserved. 
In their recent paper [7], authors of Study Group on Skill Standard for Standardization (SG-SSS) describes the reason for defining the skill set for standardization professionals as follow;

In order to collect appropriate human resources for actual tasks for standardization and to carry out effective education of standardization, it becomes indispensable

- to clarify the tasks required for standardization, and

- to clarify the skills required for the tasks.

The output of their work is the definition of 36 tasks identified for standardization, and skills defined to perform these tasks along with the three levels of skill evaluation criteria.

Task definition is done through the popular classification of de jure standards, forum/consortium standards, de facto/company-product standards and house rules, along with the following steps of standardization management:

- Strategy: strategy planning, tactics planning, founding organization, managing organization,

- Development: developing technology, developing standards, managing organization.

- Implementing: applying standards, acquiring certification.

- Promotion: promotion planning, advertising.

Skill evaluation criteria are categorized either on performance or on capability.

There are 4 aspects for performance evaluation: Responsibility, Experience, Achievement, and Contribution. And the followings are the aspects of capability evaluation: Business comprehension, Communication, Negotiation, Planning, Leadership, Presentation, Technology, and Operation.

The skill levels are the following three levels: Low for trainee, Middle for autonomously acting person, and High for leading figures instructing others as well.

So, the total descriptions are summed up around 1,200 detail description to cover the entire skills for standardization professionals. However, even in the midst of compiling these documents with Japanese experts, there emerged some criticism for this scheme of skill definition.

The major problems lie in the classification of tasks. It should be noted that the task is not classified from the standard/standardization itself but the management of standardization from for-profit corporation. 
The following Figure 1 shows the life cycle of standards. Here, we cannot see the task for strategy, implementation and promotion. They are part of the standardization management task for for-profit corporation as shown in Figure 2.

One criticism comes towards this prerequisite of the skill, that is, for the activity for "for-profit corporations." Their target is for business. We will discuss further on this point, but the argument goes that there are non-profit

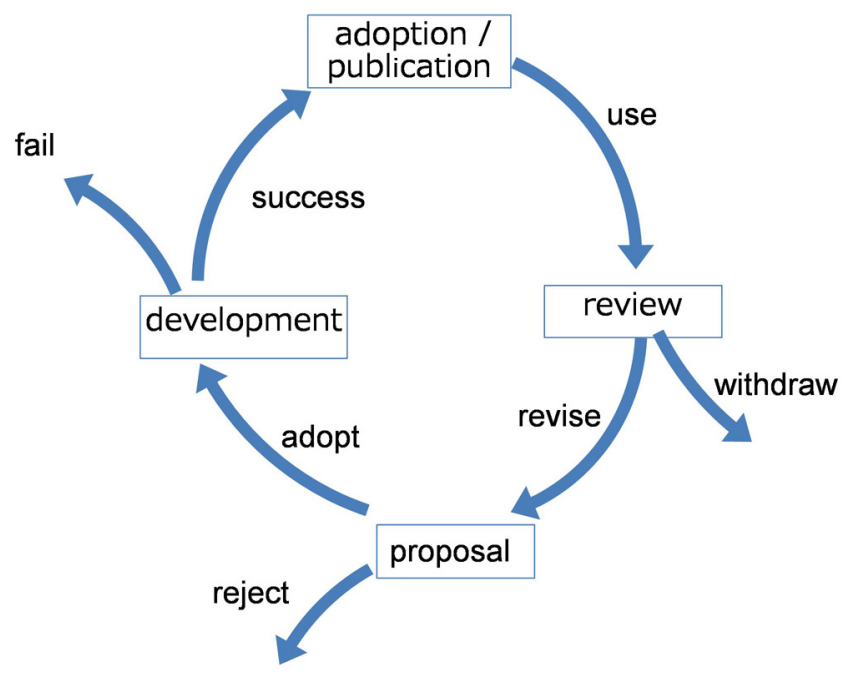

Figure 1 Standards life cycle

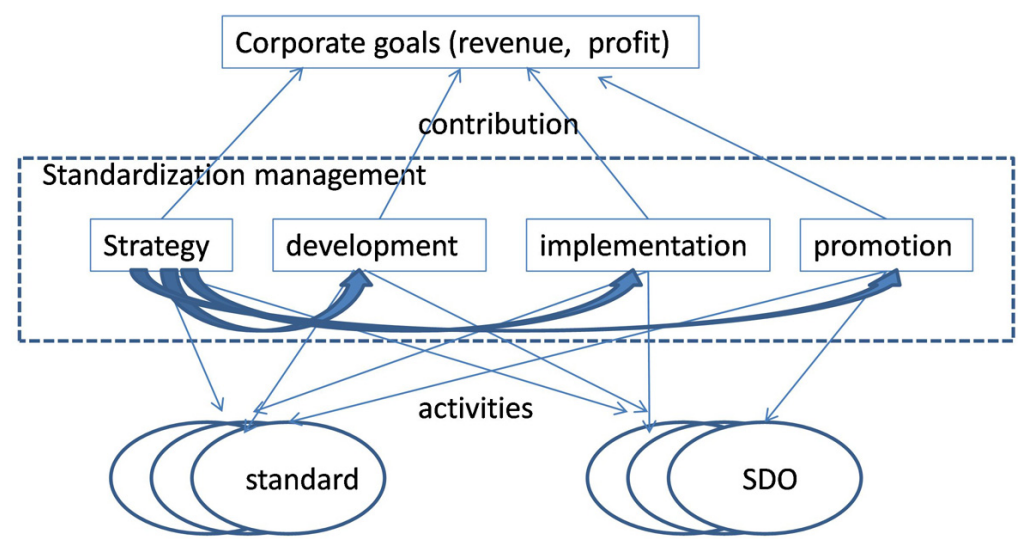

Figure 2 Standardization management tasks for "for-profit corporation" 
organizations that are dedicated for standardization, typical examples are ISO, IEC, and ITU, the Standard Development Organizations (SDOs), and the skills for those people in these organization require different skills. Both people may share the same kind of skills, but non-profit organizations' people cannot target for business.

Moreover, there are some aspects for standardization professionals who need to consider the public wellness other than the profit/merits for their company. This aspect for working for the society rather than his/her own organization is one of the great characteristic of activities in standardization, and also one of the most difficult part to be appreciated within the "for-profit organization." We will discuss this aspect further in the next section.

The other problem has come from the fact that the skill set among de jure, forum/consortia, and de facto do not differ much. Major parts of the skill set are common among them, which might be reasonable because nowadays lots of forum standards are converted into the de jure standards, and in a way, they differ only in the decision process and/or certification.

We need some other approaches to tackle this problem to classify tasks for standardization. One suggestion has been given to utilize evolutional model for standards which has been developed by Ken Krechmer [2]. His model is based on the main feature of standard: symbols, measurements, similarity, compatibility, and adaptability. Just like patents, this model describes the differences on standards, or what aspect of products and/or services is standardized, and in terms of standardization, the person in charge should have the skills specific to that kind of standardization.

It is also noted some researchers do not distinguish de jure standardization from forum/consortia standardization. For example, Büthe \& Mattli [5] categorize them both in Standardization by Private Agencies without Market Mechanism.

We will discuss the evolutional or feature model for task classification of standardization. Of course, there would be yet other approaches for classifying and defining tasks for standardization.

\section{Standardization for Public}

In 2006, IEC has published a web book entitled "Standards for business" [3] which has two subtitles: "International Standardization as a Strategic Tool," and "How companies benefit from participation in international standards setting." It is also very interesting when you try "standards for public" in 
search engines such as Google, you have no exact hit for this inquiry while the "standards for business" will get more than 2.6 million hits.

This typically shows how the standards are considered important in business. And also it shows standards are not discussed much in the context of public wellness. However, this raises a key question that the standards has been important mainly for public reasons from the historical perspective. For example, United States Standards Strategy [4] by ANSI cites John Quincy Adams' statement in 1821 to tell the basic standards (in this case, weights and measures) are "the necessaries of life to every individual of human society. They enter into the economical arrangements and daily concerns of every family."

Recent book on standardization [5] also argues for standardization mostly from economical viewpoint, however, it also cites the issue on public interest raised by European consumer group that the international standardization does not well respond to the consumers' concerns.

\subsection{Tasks for Standardization for Public}

There are many non-profit organizations engaged in standardization. Typical examples are ISO and IEC that are major international standards development organizations, although in strict sense, they are both in private agencies in contrast to the ITU which is an international governmental organization under United Nation.

People under these organizations are, even though they may be borrowed from for-profit corporations, working standardization from these organizational viewpoint, that is, for public not for profit. Governmental and/or non-profit standardization organizations are also standardizing for public. Even though some of these organizations have the mission to help for-profit corporations in standardization, the performance measurement cannot be the profit.

Also, those standardization professionals within for-profit corporations may also have their mission for standardization bodies, where their expected achievement will be primarily on the standardization activity itself. As they belong to a for-profit corporation, they share the concern of the for-profit corporation but their role in standardization may not directly produce the profit of their employer.

Even though the standardization they are involved is evaluated in the for-profit corporation's strategy for business, the proposed standard itself must have the aspect for the public good. Otherwise, no other people/groups agree with the standardization. 
Now the question comes to the point where how much of those tasks for standardization for public differ from tasks for standardization for for-profit corporations. Tasks can be categorized by the strategy/goal related ones and the operational ones which may be derived from the strategy/goals.

Clearly the goals are different for public needs and for for-profit corporation's needs. Then, also, the stakeholders may differ. For example, standardization for public needs to involve users of standards, especially consumer groups, and also needs to work with government and regulation agencies for public wellness. In the case of standardization for private interests, those consumer and government groups are the secondary audience, and this situation has been sometimes criticized.[5]

We will further investigate in two cases: one for those who work in non-profit organization and those who are employed in for-profit corporations but their primary job is for non-profit operation.

\subsection{Tasks for Standardization Professionals Working in Non-Profit Organization}

Entire tasks for people under non-profit standardization organization can be labelled differently from those working for for-profit corporations. However, most of the tasks for strategy can be applied both for non-profit and for-profit organizations. The Japanese skill standards [1] show clearly the common tasks such as: Strategy planning for standardization, Information collecting/analyzing/evaluating and tactics planning, Supervising, and Liaison establishing. Yet, the details, for example, of Liaison establishing is "lobbying activities with government and standardization organizations are performed to share information and to establish a close liaison-ship with them" are quite different in the non-profit organization. It would be more coordinating activities with other non-profit organization and/or governmental agencies. No lobbying should be necessary.

Major differences may occur in the Development tasks because these Standards Development Organization itself do not produce standards but coordinating SCs and TCs and networking Professionals and Specialists all over the world, so that they can produce standards documents.

Implementing and Promotion tasks also might be quite different. In the case of governmental organization, both tasks can be similar to for-profit corporations, but the non-profit non-government organization may not have the capability of implementation but only for helping others to implement. The objective of promotion would be different from the for-profit organization. In 
the case of non-profit organization, the promotion could be more general than the specific standards.

\subsection{Tasks Migrated in Standardization Professionals Hired in For-Profit Corporations}

This is one of the most intrigued and also most important aspect of standardization task itself. In Japan, Skill Standards are defined in some areas, notably in Information Technology (IT) [8] and in Intellectual Property Rights (IPR) [9]. Their skill sets and tasks are simple compared with the tasks for standardization professionals. Also this is more true for those experts working for non-profit standardization organization while hired by for-profit corporations.

IT Skills are for doing some IT projects covering strategy setting and maintenance. IPR Skills are also same kind and for specific for-profit corporations to deal with their own intellectual properties.

In the case of standards, the standard, even if it was drafted within the for-profit corporation for their profit maximization, does not belong to the corporationunless they are de facto standards. Lots of standardization professionals in for-profit corporationsare working for SDOs (Standards Developing Organizations) and managing both for their employers and for standardization communities.

Lots of literatures are devoted for standardization strategies for business, but they do not talk about the standards/standardization for public, perhaps because that is not their interest. Standards and standardization is among the many tools for successful business. It may also because they do not recognize the standards especially de jure standards are essentially for public. Their general attitude is to treat a standard like a patent. If you make the standard, you won it, and you can control the market and your business will prosper, just like you have got a patent registered, and control the market and your business is secured. Unfortunately, this is not true for standards. That is not the way standards are made and accepted, even though there are business aspects around the standards/standardization. Some companies utilize standards/standardization effectively so that their business can be sustainable, yet the standard itself does not belong to the company, that is quite different from the case of intellectual property.

This is also the reason behind the difficulty of evaluating standardization professionals in for-profit corporations, who work for activities of SDOs as board members, conveners, committee chairs and committee members. Their manager may tell the standardization professionals quite frankly, "why I must 
evaluate you highly, while you are not directly working for our company but for the non-profit organizations." IPR experts may not face this kind of dilemma.

It is really important for these standardization professionals to balance their activities between for-profit and for-public. Sometimes they are lucky enough to enjoy the case where their effort for the standardization for public will promise your employer a good business, however there are cases that many people cannot see why the professional's effort for standardization contribute the company's business, its sales and profit.

SDOs have paid a lots of efforts to validate the economic benefits of standards/standardization. Lots of teaching materials are developed for this purpose, visit ISO repository of teaching materials (http://www.iso.org/iso/home/standards/standards-in-education/education materials-higher-edu.htm), for example.

While the benefit of standardization is validated, the question would come who should pay the all the burden for standardization. So far, the national government and industry has paid the fee, but consumers group can tell that the consumers and people in general has paid the fee through tax payment and consumption of goods and services provided by industry. The problem is that how we can divide the benefits for each stakeholders and distribute the burden to each sector.

And, moreover, the person in the for-profit corporation who works for standardization community has various tasks that may directly related to the business and also may have no direct relation to business yet indispensable to the standardization organization.

We need more detailed analysis for the skills in this area and to search for a good evaluation system for these skills.

\section{Task Classification Based on Evolutional Stages of Standards}

One of the key question about the classification of standardization tasks is how much technical skills are needed. While each technical standards need technical skills in each area such as IT, mechanical engineering, chemical engineering and so on, how much technologies are involved in standardization in general, is the big question.

When Japanese group adopted the traditional categorization of de jure, consortia/forum, de facto, in-house standardization, it is questionable if there emerge any technical differences between these categories in technical skill 
aspects. As you see the outcome of their effort [1], there are no big differences in terms of technology, or technical skills needed.

Of course, there will be classification based on the area of technology: electronics, chemical, civil engineering and so on, however, these are just technologies that each TC (Technical Committee) or SC (Sub Committee) are dealing with. And these technical skills are regarded to belong to its own profession or business, not for standards/standardization per se.

\subsection{Krechmer's Evolutional Stage Model}

Ken Krechmer has proposed evolutional stage of standards [2] which is a reasonable model for technical classification for standardization tasks. With his model, standards are classified in the following stages: symbols, measurements, similarity, compatibility, and adaptability.

They are summarized in the following table:

Here, the symbol standard is the standard for letters and symbols, which is one of the oldest standard but also active in recent days such as IEC 60417, symbols for use on equipment. In this class, the task for standardization include the IPR related on the registration of these symbols, as well as some knowledge of linguistics, psychology, and ideogram and graphic processing.

The measurement standards are in part of metrology and quite different from symbol standard. Nowadays, the measurement standards are very technical and the Nobel prize has been awarded to the recent work [10]. Standardization skills in this area should be very technical, yet, it can be very political as for the United States customary units for measurements.

The similarity standards are attributed as the outcome of industrial age, most noted as the interchangeable parts such as screws and nuts. Krechmer describes this as follow: "Similarity standards, including process standards, safety standards and quality definitions, define the minimum admissible attributes." This kind of standardization can be possible after the mechanical

Table 1 Krechmer's Evolutional Stage Model

\begin{tabular}{lll}
\hline Standards & Ages & Major technologies \\
\hline Symbols & Hunter Gatherer & Communication \\
Measurements & Agrarian & Metrology \\
Similarity & Industrial & Interchangeable \\
& & (admissibility) \\
Compatibility & Information & Interface \\
Adaptability & Post-information & Adaptable interface \\
\hline
\end{tabular}


production of parts, and the establishment of assembly process of products based on these interchangeable parts. For this kind of standardization, you should have skills related to the production and quality control.

The compatibility standards come next, which handles interface standards such as WiFi, the cellular air interface, the Universal Serial Bus (USB 2.0), and Windows ${ }^{\mathrm{TM}}$ Applications Program Interfaces (APIs). Krechmer claims that "Standardization of similarity [...] reduces variation and therefore reduces potential innovation. However, the standardization of compatibility increases variation and innovation." Typical example are those new communication industry based on internet protocol.

As with similarity standards, compatibility standards need technical skills to design the interface, and moreover the skills for setting standards. Even though the similarity standards need technical skills and similarity standards are different from the specification of the products themselves, it is more true for interface standards. Also, interface standards may handle patents issue, in the typical case, it will come with the style of patent pool.

Thus, the standardization skills for compatibility standards may span a lot from the purely technical skills to understand the essence of communication protocol to the legal aspect of patent pool, as well as grouping and business management skills for establishing new industry.

The last category Krechmer proposes is the adaptability standards. This is an adaptable interface standards, targeting multiple interface standards co-exit as long as they are adaptable. Typical example that Krechmer refers is the G3 fax machine protocol. Within this framework of adaptability standard, vendors and users both can enjoy the benefit of innovations while retaining the basic services of communication.

For this adaptability standards, the key technical skill is with etiquettes [11] that will enable each interface to be adaptable with the other standards. On the other hand, the adaptability standards are expanded from compatibility standards, and the skills for compatibility standards will be required in this kind of standards as well.

\section{Task Classification for Open and Management Standards}

There would be other classification approaches than those in preceding sections for standards/standardization as well. One approach would be Open Standards and Open Standardization. This highlights traditional standard and standardization to be called as Close Standard and Close Standardization. 
While the definition of Open Standards and Open Standardization varies from people to people, their key gradients would lie upon their process of making (and/or defining) standards and also for the availability of standards.

Their underlying technology is the Internet and open collaboration is the feature of their process.

The other task classification for standard can come from the so-called Management Standards, or Management Systems Standards, which is quite different from the traditional technical standards where the organization's management process is standardized. ISO describes this as "They provide a model to follow when setting up and operating a management system" [13]. Some people classify the traditional standards as technical standards to highlight the differences from these management standards.

However, those Open Standards and Management Standards are less connected technologies than those Krechmer's evolutional model addresses.

\section{Summary}

In this paper, we have explored non-traditional classification of standardization tasks. This work has been triggered from the Japanese study on skill standards for standardization professionals [1]. Their study has adopted traditional classification with de jure, forum, de facto, and in-house standards along with the processing stages of Strategy, Development, Implementing and Promotion.

Since this traditional classification for standardization tasks is not enough for handling all the tasks for standardization, we have studied several approaches in this paper. These approaches are not exhaustive, so there would be still other approaches as well. We would like to further explore and discuss those new possibilities.

However, it can be noted that the Standardization for Public should have an impact on standardization in the near future, since the voices for promoting standardization for public has becoming popular in recent ICES 2013 Workshop [12] held in June 2013. These voices are high especially for under development countries where farmers, fishermen and small merchants have the strong concern with the standards but cannot have the luxury to own their standardization professionals but only can rely to the standardization staff within government and public service.

Task classification based on Krechmer's Evolutional Stage Model is also important in the sense that these shed some lights on technologies behind the standardization so that the university level curricula can be organized along this set of technologies. 
Also more work can be employed for other approaches such as the Open Standards/Open Standardization and Management Standards. These are rather new to the standards/standardization so we may need more experiences with these approaches.

\section{References}

1. Skill standard - Evaluation for skills of human resource required for standardization, Ver. 1.03, 2013-05-31, http://docbox.etsi.org/workshop /2013/201306_ices/presentations/7-papers\%20and\%20posters/kurokawa \%20et\%20al\%20iieej\%20japan\%20skill\%20standard.pdf 2013.

2. Ken Krechmer, "Balanced Standardization" Journal of IEEJ, Vol.41, No.5, pp.568-574, http://www.y-adagio.com/public/committees/std/ serial_sv/st4.pdf.

3. Henk J. de Vries, Standards for business - How companies benefit from participation in international standards setting, IEC Centenary Challenge 2005.

4. ANSI, United States Standards Strategy, Third Edition, ANSI, 2010.

5. Tim Büthe \& Walter Mattli, 'THE NEW GLOBAL RULERS - The Privatization of Regulation in the World Economy', Princeton University Press, 2011.

6. APEC Sub-Committee on Standards and Conformance, 'Education Guideline 3: Textbook for Higher Education - Standardization: Fundamentals, Impact, and Business Strategy', APEC, 2010.

7. Toshiaki Kurokawa et al., 'Skill standard -Evaluation for skills of human resource required for standardization', Proceedings of ICES Workshop 2013, 2013.

8. IPA, The Skill Standards for IT Professionals, 2008, http://www.ipa.go.jp /english/humandev/forth_download.html

9. METI, The Skill Standards for IPR Human Resources, 2007, http://www.meti.go.jp/policy/economy/chizai/ipss/(in Japanese).

10. Quantum Information Science and Engineering Program at NIST, http://www.nist.gov/pml/div684/qip.cfm

11. Krechmer, K.: The fundamental nature of standards: technical perspective. IEEE Communications Magazine, 38(6), pp. 70-80, 2000. Also available at http://www.csrstds.com/fundtec.html 
12. ICES and WSC Academic Day 2013, held at ETSI, June 2013. http://www.etsi.org/news-events/past-events/658-2013-ices-wscconference

13. Management system standards. http://www.iso.org/iso/home/standards /management-standards.htm.

\section{Biography}

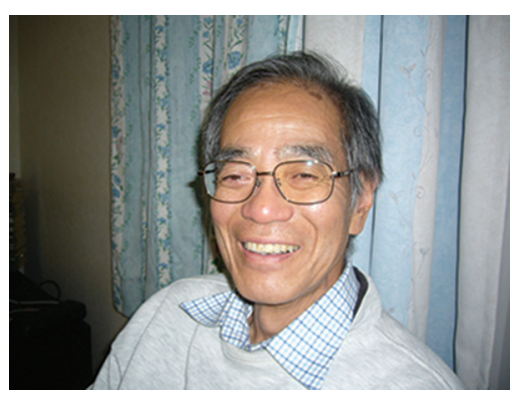

Toshiaki Kurokawa is an ICES Founder and now self-employed as an independent consultant to manage Design Thinking Research \& Education at his home. With his colleagues, he formed a joint Study Group on Skill Standard for Standardization (SG-SSS) under IEEJ and IECEJ. At IEEJ, Mr. Kurokawa is the chairperson of SIG on Education about Standardization. He is also an

Affiliated Fellow at Science and Technology Foresight Center, National Institute of Science and Technology Policy, Ministry of Education, Culture, Sports, Science and Technology (NISTRP under MEXT) in Japan. He was a Researcher at Intellectual Property Science Laboratory of Kanazawa Institute of Technology, located in downtown Tokyo before February, 2013, where he was engaged in the project of "Evaluation for skills of human resource required for standardization" sponsored by Ministry of Economy, Trade and Industry (METI) in Japan. Mr. Kurokawa has been engaged in standardization through his engineering/marketing/management career at IBM and SCSK (formerly CSK) Corporation from around 1990 mostly in ICT area. In 2006, he founded International Cooperation on Education about Standardization (ICES, http://www.standards-education.org/) with his friends, and has talked his experience at various places including foreign countries such as Indonesia, Malaysia, and USA. He has published numerous papers on education about standardization, ICT and Design Thinking at such journals as "Science \& Technology Trends - Quarterly Review." His research covers other areas such as Requirement Engineering, Software Testing, Ontology and Cloud Computing. He is also working for Kids, Their Future \& Design for K-6 children, and Senior-Junior Cooperation Initiative to re-vitalize senior experts. 
